\title{
Challenges in Providing Surgical Procedures During the COVID-19 Pandemic: Qualitative Study Among Operating Department Practitioners in Pakistan
}

\section{Sara Rizvi Jafree}

Forman Christian College

Ain ul Momina

King Edward Medical University

Nudra Malik

Lahore College for Women University

Syed Ashgar Naqi

King Edward Medical University

Florian Fischer ( $\nabla$ florian.fischer1@charite.de)

Charité - Unversitätsmedizin Berlin https://orcid.org/0000-0002-4388-1245

\section{Research}

Keywords: Emergency surgery, coronavirus, operational department practitioners, patient safety, public health sector

Posted Date: December 31st, 2020

DOI: https://doi.org/10.21203/rs.3.rs-136793/v1

License: (c) (1) This work is licensed under a Creative Commons Attribution 4.0 International License. Read Full License 


\section{Abstract}

Background: Operating Department Practitioners (ODPs) are neglected human resources for health with regard to both professional development and research for patient safety. The surgical theatre is associated with the highest mortality rates and with the onslaught of the COVID-19 pandemic. ODPs are key practitioners with respect to infection control during surgeries. Therefore, this study aims to describe challenges faced by ODPs. The secondary aim is to use empirical evidence to inform the public health sector management about both ODP professional development and improvement in surgical procedures, with a specific focus on pandemics.

Methods: A qualitative study has been conducted. Data collection was based on an interview guide with open-ended questions. Interviews with 39 ODPs in public sector teaching hospitals of Pakistan who have been working during the COVID-19 pandemic were part of the analysis. Content analysis was used to generate themes.

Results: Ten themes related to challenges faced by ODPs in delivering services during the pandemic for securing patient safety were identified: (i) Disparity in training for prevention of COVID-19; (ii) Shortcomings in COVID-19 testing; (iii) Supply shortages of personal protective equipment; (iv) Challenges in maintaining physical distance and prevention protocols; (v) Human resource shortages and role burden; (vi) Problems with hospital administration; (vii) Exclusion and hierarchy; (viii) Teamwork limitations and other communication issues; (ix) Error Management; and (x) Anxiety and fear.

Conclusions: The public health sector, in Pakistan and other developing regions, need to invest in the professional development of ODPs and improve resources and structures for surgical procedures, during pandemics and otherwise.

\section{Background}

During the COVID-19 pandemic, the healthcare sector has been facing some of the biggest challenges ever with regard to service delivery and patient safety [1]. Deciding to continue emergency surgeries during a pandemic while maintaining standards for efficiency in both surgical care and infection prevention has not been uncomplicated. Errors in the hospital occur more frequently in the operating theatre than in any other clinical setting $[2,3]$. The quandary of contemporary times is that both patients and practitioners during emergency surgeries are at threat from multiple risks of: (i) COVID-19, (ii) hospital-acquired infections, and (iii) surgical site infections [4]. Surgical site infections are more common in lower and middle income countries due to poor infection control practices, shortage of resources and poor management [5]. Contracting an infection generally has risks of morbidity and mortality, but in times of COVID-19, there are additional fears. These fears relate to contracting the virus, remaining uncertain about recovery, spreading the disease to family members, having to isolate or getting admitted to an intensive care unit, and losing income or employment [6]. 
Until now, there is little research about Operation Department Practitioners (ODPs) globally [13]. Historically, the ODP role was created as an additional support resource for the theatre nurse [14]. From hospitals records of the eighteenth and nineteenth centuries, ODPs were classified as 'handlers' of patients or 'box carriers' for surgeons [15]. The role originated without requirement of specific skills or qualifications, with demand for undefined assistance in the surgery, mostly with respect to cleaning, carrying patients, and supporting skilled staff. Later on, responsibilities of anaesthesia were added and some level of training helped to cement the ODP role as assistants in the operating theatre [16]. Even today, across nations and hospital organizations, there is disparity about the exact role and responsibilities assigned to ODPs. In some settings, their work is confined to the role of technicians and setting up of equipment, and in others their roles are indistinguishable from that of theatre nurses [17]. ODPs are also responsible for the sterilization and disinfection of the theatre, theatre equipment, and theatre instruments; making them important team member in times of pandemics.

One of the weakest knowledge areas globally is the investigation of the culture and behaviour in the theatre and the experiences and challenges faced by ODPs [24, 25], with no research from Pakistan [23]. We believe the strength of interviewing ODPs enables (i) the identification of challenges and non-medical factors that cannot be found in textbook material, because they are embedded in local and regional issues involving organizational culture and interpersonal relations, and (ii) the inclusion of narratives by a neglected and marginalized practitioner group who have critical knowledge about challenges in the theatre as they perform multiple roles and deal with other practitioners in the theatre and hospital.

In this study we aim to identify the challenges faced by ODPs, as perceived and experienced by them. Furthermore, we aim to answer the question which improvements are needed to secure patient safety measures for emergency surgical teams. Our secondary aim is to use empirical evidence to inform public health sector management about both ODP professional development and improvement in emergency surgical procedures, with a particular focus on the COVID-19 pandemic.

\section{Methods}

\section{Study design}

A purposive qualitative design was adopted for this study to interview ODPs. We used a multistage sampling process (Table 1). Our selection criterion for hospitals was public sector teaching hospitals, which had operational emergency theatres during the COVID-19 pandemic. We used a list provided by the Punjab Healthcare Commission, which classifies teaching hospitals under category 1 group. All these hospitals are run by the Specialized Healthcare \& Medical Education Department, Government of Punjab. From a list of 23 such hospitals [26], we received a positive response from 9 hospitals who provided contact lists of their ODPs. Our selection criterion for ODPs was those who had been: (i) working in emergency theatres during the first wave of COVID-19 in Pakistan between the months of February to June 2020, and (ii) working in the theatre for more than two years. 
Table 1

Multistage sampling process

\begin{tabular}{|llll|}
\hline Stage & Sample & Selection criteria & $\begin{array}{l}\text { Final } \\
\text { sample }\end{array}$ \\
\hline 1 & $\begin{array}{l}\text { Hospital selection, based on } \\
\text { permission from provincial } \\
\text { government }\end{array}$ & $\begin{array}{l}\text { Public sector teaching hospital, with } \\
\text { operational emergency theatres during } \\
\text { coronavirus pandemic }\end{array}$ & $\begin{array}{l}\text { 9 hospitals } \\
\text { from Punjab } \\
\text { province }\end{array}$ \\
\hline $\begin{array}{l}\text { Recruitment of ODPs, based } \\
\text { on contact provision by } \\
\text { sampled hospitals }\end{array}$ & $\begin{array}{l}\text { (i) ODPs who have been working for more } \\
\text { than 2 years in government hospitals } \\
\text { (ii) ODPs who have been working in months } \\
\text { of February 2020 to July 2020 during the } \\
\text { coronavirus pandemic }\end{array}$ & 39 ODPs \\
\hline
\end{tabular}

\section{Interview guide}

We used an open-ended interview guide (Supplementary Appendix 1) which has been developed for this study, asking the following four broad questions:

1. Have you received any training and guidelines for management during COVID-19?

2. In the current COVID-19 scenario: What are the problems and challenges faced by the surgical teams?

3. Would you like to share your experiences of any safety risks at work?

4. What do you think can be done to avoid violations and medical errors to improve patient safety?

We also used prompts, derived from WHO [27], to support ODPs in understanding the area of questioning and giving relevant answers to the study area [28]. These prompts related to the following issues:

- Communication with the patient and the family

- Inter-provider communication

- Distractions

- Care coordination

- Access to necessary information

- Education/training

- Blood transfusions, specific tasks in operating theatre

- Labs, studies, testing

- Equipment/physical environment

- Example of adverse events

- Staffing

- Transitions/handoffs 
Although English is the official working and study language in Pakistan, the interview guide was translated to Urdu. This was done to facilitate ODPs who are not necessarily all highly educated and to provide convenience in answering questions in the native language (Supplementary File 1).

\section{Data collection}

In total, we gained a contact list of 54 ODPs. We first text messaged them, requesting them for permission to participate in the study. In case of no reply, we followed up with one more text message. Overall, we received a reply from 43 ODPs showing willingness to participate in the study. Finally, we were able to complete interviews with 39 participants. Telephone interviews took place between July and August 2020. Audio recording of the telephone interviews was necessary to allow for a subsequent data transcription.

\section{Data analysis}

At first step, data was transcribed from the audio interviews by one researcher and afterwards checked and confirmed by two further researchers. This process starting already after the first interviews had been conducted. NVIVO software was used for generating themes and sub-themes [29]. Thematic content analysis was used to develop themes and sub-themes [30]. Independent manual theme generation was conducted by the first three authors to confirm themes and ensure nothing was excluded. Examples of theme generation and coding have been provided in Supplementary File 2. After the 33rd interview, authors agreed that saturation point had been reached [31], because no new topics emerged. We discussed the findings with three ODPs, who had not participated in the study and two health administrators to confirm the results.

\section{Results}

A considerable number of ODPs received only secondary education (38.4\%), while the majority have an Operational Theatre Technician Diploma of two years (53.8\%). Most interview partners (IP) $(69.2 \%)$ were earning a monthly salary between USD 141.7 and 209.5, and the rest (30.8\%) earned up to USD 338.9 per month (Table 2). 
Table 2

Socio-demographic characteristics of participants $(n=39)$

$\begin{array}{ll}\text { Variable } & \mathbf{f}(\%) \\ \text { Age } & \\ 21-30 \text { years } & 13(33.3) \\ 31-40 \text { years } & 14(35.9) \\ 41-50 \text { years } & 12(30.8) \\ \text { Last degree } & \\ \text { Matric (10th grade) } & 7(17.9) \\ \text { Intermediate (12th grade) } & 8(20.5) \\ \text { Operation Theatre Technician Diploma } & 21(53.8) \\ \text { BSc in OT Technology } & 3(7.7) \\ \text { Years of service } & \\ 3-5 \text { years } & 9(23.1) \\ 6-15 \text { years } & 18(46.2) \\ 16-35 \text { years } & 12(30.8)\end{array}$

Monthly salary

PKR 23,000-34,000 (USD 141.7-209.5)

$27(69.2)$

PKR 35,000-55,000 (USD 215.7-338.9)

$13(30.8)$

All PKR to USD conversions of this study have been done at the rate of 1 USD $=162.805 \mathrm{PKR}$, as at 17th October 2020, using online currency conversion information from: https://www.xe.com/currencyconverter/convert/?Amount=490\&From=PKR\&To=USD

The ten themes with respective sub-themes are described below.

\section{Disparity in training for prevention of COVID-19}

There was a persistent belief in ODPs that they did not require any additional training for COVID-19 prevention. A difference in this judgement existed between participants in terms of having received no training versus having received some briefing for COVID-19 prevention and management. ODPs who confirmed they had received some briefing, shared that the instructing had come from senior colleagues. However, two ODPs from different hospitals did mention that their briefing had come from a designated infection control nurse. Overall, ODPs highlighted that due to a lack of training they were dependent on 
learning more about the disease and getting updates on prevention from the media and on-the-job experience.

\section{Shortcomings in COVID-19 testing}

All ODPs confirmed that in their line of work and emergency services, time for COVID-19 test results was not possible. ODPs shared opinions and misconceptions about testing and safety precautions. Of concern was that an ODP believed that if the Antibody (IgG) test was clear, it meant they did not have to take strict precautions:

"If the lgG is clear will do not have the pressure to take so many precautions." (IP3)

Despite international consensus that safety protocols should be managed consistently across all patients, an ODP shared his belief that it was unfair to treat all patients in the same way:

"It is not fair to suspect every patient! Treating all of them as COVID-19 patients and taking strict precautions is humiliating for them." (IP17)

Most ODPs were not being supported by their employer for regular testing or healthcare consultancy at their hospital. One ODP shared the burden they faced in paying for the test themselves and seeking consultancy for symptom management:

"About a month ago, I was not feeling well. So, I asked the doctor in my hospital to check me. But he did not even touch me after hearing about my symptoms. He told me to go to the expo [testing centre] and that is where my test will be conducted. I could not go to the expo as it was too far. Instead, I paid PKR 6,000 [USD 36.94] at a private lab and got the test done from there." (IP31)

\section{Supply shortages of personal protective equipment (PPE)}

All sampled ODPs confirmed that they had never received N95-masks and that there were shortages of surgical masks and gloves. Protective gears have even been reused due to shortages:

"We have never received N95 masks. There are not enough supplies of surgical masks and gloves. Due to shortages we sometimes sterilize surfaces with one glove." (IP20)

Furthermore, maintaining safety rules of changing protective gears was not possible due to shortages:

"We do not have enough resources to follow everything according to rules for safety. It is very difficult. If there are no new masks, we clean the ones we used earlier with chlorine and then wear it." (IP21)

In addition, the quality of mask supplies has been criticized:

"The masks that we have been provided are of extremely poor quality. The quality was so bad that it becomes difficult to breathe once they are worn." (IP9) 
All ODPs stated that they - along with other allied health professionals (AHPs) - were not supplied with PPE kits, and were expected to use either disposable gown-like aprons or their old cloth gowns:

"Sometimes we get a disposable apron. But mostly we use our old cloth gown." (IP36)

An ODP shared why he believed that his profession and other AHPs were at higher risk of exposure:

"The ones that are being affected the most are us [the ODPs] and others like ward boys, cleaners, hospital security, paramedics, and receptionists. We have more interaction with the patients and family attendants... We are at the frontline. But we are not provided the entire PPE kit." (IP27)

The problem of supply delays and discriminatory distribution is due to external suppliers and hospital administration, respectively:

"The external supplier does not deliver the PPE kit on time. When they finally reach the hospital, they are provided to some VIP patients and senior healthcare practitioners, but never to us [the ODPs] or the paramedical staff." (IP22)

\section{Having to purchase PPE or ask family attendants of patients to buy it for them}

Many ODPs bear the burden of having to purchase PPE kit themselves. An ODP confirmed his commitment to bearing expenses and continuation of services for emergency patients despite shortage of supply from employer:

"Some of us have purchased our own equipment, like glasses and N95-masks. We can't put a condition that we'll only work when administration provides us with PPE kit." (IP10)

Sometimes even family attendants of patients are asked to purchase protective gear for them:

"Even right now, the mask that I am wearing, I bought it with my own money. Sometimes due to lack of time, we ask the attendants of the patient to purchase masks and gear from outside and bring it to us." (IP20)

\section{Challenges in maintaining physical distance and prevention protocols}

\section{Inability to maintain physical distance from patients and co- workers}

All interviewed ODPs confirmed that they were unable to maintain physical distancing from patients and co-workers. An ODP shared how patients in emergency usually cannot wear masks due to breathing difficulty or general anxiety: 
"Patients in emergency situations are agitated and in anxiety. Many have breathing problems, so they can't wear masks or keep them on. For basic intimate intravenous line, catheter placement, cardiac monitoring of the patient we cannot maintain distance." (IP15)

Another ODP shared that when they worked, ate and rested with co-workers in the common room, masks were not worn:

"We are unable to prevent physical contact or maintain distance during changing of masks and gloves, or changing of gear in changing rooms. We eat together and share one rest room [with co-workers]. And usually masks are removed during eating and resting." (IP2)

\section{Lack of time for complete disinfection between emergency cases}

All ODPs confirmed that emergency surgeries prevented complete disinfection of the theatre between cases. One ODP highlighted what disinfection and sanitization protocols were manageable in 15 minutes:

"If the burden of number patients is a lot and there's no free time in the theatre, then we just change our caps, mask, and gloves. If there is any smearing, like the patients' blood falls, the sweeper is called to clean this. We clean the instruments and machines. This is all that can be managed in 15 minutes." (IP1)

Another ODP explained the theatre sterilization process in his hospital:

"The theatre is carbolyzed twice every day. In between patients we turn on the gamma lights." (IP11)

\section{Disinfection protocols same as before versus improved after COVID-19}

Divergent pathways for disinfection of theatre across hospitals after COVID-19 are described by the study participants. One ODP confirmed that the sanitation process has not changed:

"We follow the same procedure for sanitation as we did in the past [before COVID-19]. First, we spray chlorine, then we use sanitizer and mostly we do scrubbing with pyodine. We do this twice a day." (IP2)

Another ODP stated that they were more careful now and different cleaning agents are used separately to ensure complete sterilization:

"We are much more careful now. Instead of cleaning the theatre with just chlorine, we also use Dettol spray separately. We also use Phenyl separately. This way three cleaning agents are used and the theatre is cleaned thoroughly after each patient." (IP4)

\section{Human resource shortages and role burden Shortage of ODPs}


All men that participated in the study complained about shortages in staffing of ODPs, before and since COVID-19. One participant lamented about the need for more ODPs in each shift and having to work two shifts in a day:

"We have six theatres in the operation floor upstairs and six OTAs [Operating theatre assistants] on duty in one shift. At one time, all six theatres may be in use. We need more than one OTA for each theatre." (IP11)

Another participant highlighted that in his hospital, since the COVID-19 outbreak, each ODP was expected to manage two surgeries simultaneously:

"There are four theatres and only two OTAs in each shift. They [hospital administration] have limited the numbers of OTAs due to COVID-19. Sometimes we have to take care of two surgeries at one time and run between cases." (IP13)

Some shared that if they were infected by COVID-19 but did not have symptoms, the administration compelled them to come to work due to shortages of ODPs:

"There is some staff here that caught COVID-19 and they submitted an application for leave. However, as they had no symptoms, the administration asked them to wear a mask and come to work. They were warned that if they did not come, their salaries would be stopped." (IP20)

\section{Added burden and time required for prevention}

Participants complained about the additional time and energy required for preventive measures. Safety routine at work and home have been described as both become time-consuming and fatiguing:

"There is a lot more that needs to be done now - with regard to sanitation and masking. It is tiring and cumbersome." (IP1)

Another ODP gave an example of the exhaustion associated with separation and washing of clothes:

"There are many things that have changed in our lives, requiring more time and effort. We cannot wear the same clothes consecutively for two days. We have to remember to change and wash our clothes as soon as we go home. As we are always very tired, sometimes we just leave them [clothes] under the sun." (IP21)

\section{Additional role allocation without compensation}

ODPs complained about how they were now tasked with more work related to disinfection and sterilization of theatre, which was not part of their original job profile. A participant shared how they were now doing the work of the sweepers and janitorial staff without any compensation:

"All of this sterilization should be done when we arrive in the morning by the sweeper and janitorial staff. It was their [sweeper/janitorial staff] job and was not a part of our routine." (IP8) 
The same ODP complained that extra work had been allocated without equitable increase in compensation:

"We have been assigned more work and without an addition in salary." (IP8)

\section{Problems with hospital administration \\ Lack of response from and discriminatory behaviour by administration}

Participants complained about the lack of response from administration and discriminatory behaviour against ODPs. The difficulties in asking for resources that were due to ODPs are brought to attention:

"The administration is very bad. We cannot say much about them. If we ask them about anything, there is no response. Since the pandemic, we have been asking them for N95-masks, but they claim they are not in stock." (IP10)

Mostly supplies went missing and when some distribution of supplies took place, doctors and senior staff were favoured over them:

"We know that deliveries of 50,000-60,000 N95-masks were received by administration. Out of 60,000 masks, only 200 were provided to the doctors and senior staff... And none to us. We still don't know, to this date, where the rest of the masks went." (IP20)

\section{Theatre design and waste disposal}

ODPs emphasized the unplanned theatre design and problems related to lack of space, no separation between entrance and exit from theatre, and insufficient separation of waiting area for family attendants. One ODP described how the theatre design prevented infection control and patient safety:

"The problem is that the theatres are congested and the entry and exit to the theatre are not separated. The waiting area for family is right outside the theatre, which is even worse. The theatre is not built in a way which facilitates infection control." (IP13)

Another ODP lamented about the lack of clear zones for sterile core and dirty outer spaces, as well as the lack of responsiveness by the government to make changes:

"We don't have a border line between clean and dirty areas. And the construction of the buildings does not allow a division. The government officials come here but they don't listen to our suggestions and they don't care about these things." (IP31)

\section{Critical obstetrics patient management issues}

Some ODPs discussed the problem related to the mandatory requirement by government hospitals for the registration of expectant mothers at the third month of pregnancy. Due to non-registration, many mothers 
travelling from remote areas and requesting admission for emergency assistance were turned away. An ODP explained how difficult it was to turn away mothers in their ninth month when they may require emergency assistance:

"Some unregistered mothers from far-off areas come for emergency deliveries. They are in distress, but if the doctor decides they are not in need of C-section [caesarean section], we have to turn them away. They beg us to register them and re-consider their C-section, but we cannot help them. It's unfair and cruel." (IP16)

\section{Exclusion and hierarchy}

\section{Governance inclusion and low salary}

All participants complained about the lack of involvement in governance matters and their inability to contribute as a team member at the hospital. They also unanimously complained about low salaries and remuneration. An ODP described how his low employment contributed to his inability to communicate with the administration or government about resource shortages or other problems:

"I am a middle-level worker, who has a job of 14 scale. I am unable to complain to either the administration or the government about short resources or any other matter. All I can do is to perform my duty and keep silent." (IP15)

Another study participant complained about his low salary and inability to demand better remuneration due to the fear of reprisal from the administration:

"My monthly salary is PKR 34,000 [USD 215.45] after 18 years of service. We have not had a promotion or salary increase in the past two years. It's becoming difficult to survive as everything is becoming expensive now. Can a poor 13 scale worker make any complaints? The administration will immediately turn against us." (IP29)

\section{Inferior subordinate relationship with doctors and nurses}

ODPs described their inferior position in a hierarchical setup, where they were considered invisible members of the team, and doctors and nurses were given preferential treatment. A participant gave the example of their inferiority in the way protective gear was distributed to doctors but not to ODPs:

"How else can we explain our invisibility? You can see the doctors have everything available. They are wearing masks, shields, and gowns. This clearly means they receive it and there is no supply issue. The issue is about seniority and exclusion. They don't forward the safety kits to people like us. We feel like crying after witnessing such injustice." (IP35)

The reporting lines for ODPs are mostly the theatre nurses, who - as one ODP described - are not always accommodating: 
"We report to the theatre nurse. If we need anything, we have to ask the sister [nurse] to arrange and coordinate it for us. Most of the time we cannot communicate with her or she is non-responsive." (IP6)

\section{Exclusion from training and skill development}

ODPs also complained about the lack of training and skill development opportunities for them in comparison to the opportunities available to doctors and nurses. A participant shared how ODPs had always been side-lined for training, whereas doctors and nurses had been receiving support for many years:

"Doctors and nurses have received special training for COVID-19, but we didn't receive any special training. We are following safety measures on our own. This is nothing new. Doctors and nurses have always received more training support, whereas we have always been considered non-skilled support staff." (IP15)

\section{Teamwork limitations and other communication issues Lack of coordination with pre- and post-operative team}

The lack of communication with the post-operative team has been described as a problem for efficacy and surgical outcomes. An ODP shared his aggravation in not being able to pass on vital information related to the patient for post-operative care:

"We are not linked with the post-operative care team. We send the patient to the post-operation after the theatre. If we have special instructions regarding the patient, we are unable to communicate it or don't know whom to share it with." (IP6)

Another ODP complained about the lack of communication with pre-and post-operative staff for patient management and handover:

"There is sometimes confusion about place and timing of transfer of responsibility. Sometimes, we have to return - after the patient is brought in the theatre - to the ward nurse to get necessary information about the patient. Sometimes the doctor leaves without communicating when the patient can leave from the surgical recovery room." (IP19)

\section{Collective training and team-building of operational theatre team}

ODPs were aware about their worth in the team and the necessity of optimal teamwork in the theatre. However, there were complaints about sub-optimal teamwork and lack of comfort and communication. An ODP shared how he believed team training workshops were needed to improve patient safety:

"In the theatre we have to be a well-tuned team. The doctors, the nurses, the paramedics, they all should help each other and work as a team. This is not happening. We need to be trained and attend workshops 
together. We need for the surgical team to have more understanding and comfort with each other." (IP9)

\section{Poor communication with patients from diverse backgrounds}

ODPs complained about the difficulties in communicating with poor and illiterate populations that visited governmental hospitals. The lack of communication between patient and provider compromises patient's safety:

"Government hospitals deal with poor and illiterate patients. We can't tell you about the problems we face with such patients: From inability to communicate with them, understand their medical history, to a lack of correct reporting of symptoms. The failure in communication influences both their treatment and recovery." (IP25)

\section{Difficult and multiple family attendants}

ODPs described the difficulty in managing multiple family attendants. Time required to deal with them takes time away from patient care. Furthermore, hostility and aggression among family attendants have been reported. In addition, there is a problem of exposure and contamination due to family attendant crowding and the lack of respect afforded to ODP's instructions for safety:

"It is common to have many family attendants just outside the theatre. We want to ask them to wear masks or use sanitizer. But only the staff nurse can talk to them. They will not listen to us, as they know we are junior staff. We sometimes have to call security." (IP7)

Another ODP highlighted the mistrust of family attendants about theatre practices and their aggression:

"Ten people come inside with one patient, without any masks. We try to tell them about precautions and danger, but they don't listen to us. They imagine all sorts of conspiracies happen within the operation theatre and can get hostile and aggressive with us." (IP36)

\section{Error management}

\section{Incapacity to share safety concerns with doctors or management}

Participants shared that they were unable to discuss theatre procedure or problems with the doctors or management. An ODP stressed that if they dared to ask a question or raise a complaint, it would be turned against them:

"How can we control safety of patients or report errors when we know about them? This is something the doctors have responsibility for. We are just minor assistants, whatever they [doctors/senior colleagues] say, we'll do it. If we try to talk to them [doctors/senior colleagues] or the management, it creates a complaint against us." (IP30) 
Another ODP revealed that their primary concern was to do their job and not getting involved in complaining or whistle-blowing:

"It is not our job to complain. And who should we complain to? The burden is high on us and we have to deal with everyone very carefully. We are doing our best given the circumstances." (IP33)

\section{Unintentional violation due to urgency and lack of time}

Participants did impart that human error was a part of surgical procedures, but that their work was especially susceptible due to having to deal with emergency cases. An ODP gave the example of a caesarean section delivery:

"Some safety breaches occur due to the hurried nature of our work. For example, the bigger problems happen with mothers who need an immediate C-section. When the heartbeat is less, we have to compromise on sterilization and urgently perform the surgery." (IP7)

Another ODP described the additional work burden during the lockdown leading to errors:

"We have been working non-stop since the lockdown... Apart from the emergency cases, even nonemergency cases are sent to us, like orthopaedic fractures, surgical drainage for head and neck infections, and cancerous tumours or fibroid removal. Mistakes are inevitable for us due non-stop work." (IP24)

\section{Breaches in protective gear management}

ODPs also described how breaches in protective gear management were inevitable due to the workload and emergency setting. An ODP shared his experiences of the slipping of masks and compromised gloves:

"You forget that we are also humans. So, mistakes are inevitable - like the slipping of masks and compromised gloves. For example, when we have to handle the theatre door and then clean the patient's hands without time in between to change gloves. But we try our best. Mistakes like this, that happen unintentionally, are a part of theatre life and surgical procedures." (IP8)

\section{Errors in communication by doctors during surgery}

ODPs shared the dilemma of not always understanding doctor instructions during emergency surgical procedures due to a lack of knowledge, training and pre-surgery communication:

"If errors happen it is often because our doctors tell us to do something in a certain way, which we have no knowledge of. Obviously, one gets confused at such a time. It is also scary because the patient's life may be at stake. We are used to all of this by now. It used to be a problem at the beginning of the career. But it is not our fault... We are very junior team members." (IP9) 
Another ODP shared that despite the lack of understanding at times they continue to perform their tasks to the best of their ability:

"They [the doctors] tell us things in the theatre, which at times we cannot understand completely. We don't question them and do the best that we can to avoid delays. This is how it is." (IP38)

\section{Work burden related errors}

Participants described the predicament of working in emergency surgeries coupled with non-stop work pressure without adequate rest days. An ODP asserted that excessive work burden made errors in the theatre a normal part of their lives:

"Don't ask us about errors. Ask us about how much we work. We have to work consecutively for six days. Without adequate rest, it is inevitable to make mistakes. Our duty days should be divided such that we work for two days and rest for one day." (IP10)

Another participant shared the amount of non-theatre work burden borne by ODPs:

"It is not uncommon for the pre-operative and post-operative staff to demand help from us. We have to run up and down to monitor pre-surgery antibiotics and many times we stay late for the post-operative support of the patient - like for femoral artery bandage removal." (IP27)

\section{Anxiety and fear}

\section{Getting infected despite following safety measures}

ODPs shared their perplexity and fear of contracting COVID-19 despite precautions:

"There is a risk - no matter how many precautions we take. This is the scary part." (IP18)

Another ODP maintained that despite maintaining safety protocols he knew of people getting infected:

"Even when some of our colleagues have been maintaining protection and safety measures, they are getting infected with COVID-19. What can we really do?" (IP1)

\section{Fear of catching COVID-19 in the theatre}

Participants shared that they were afraid of contracting COVID-19 during surgical procedures. An ODP described the fear felt in a small theatre with many people in close contact over a surgical table for long hours:

"Sometimes we are dealing with asymptomatic patients. We are 6 to 8 people in a small theatre for many hours. Obviously, we are scared that we may get it [COVID-19]." (IP12)

Another ODP shared that the fear of one member of the surgical team catching COVID-19 is always there and that there is nothing much that can be done - except to wait for symptoms to occur: 
"We are scared. We cannot guarantee that someone on the surgical team will not get it. But we keep working. If symptoms appear then we get tested. This is how it has been since February [2020]." (IP13)

\section{Fear of passing infection to family}

Most study participants shared their major apprehension about passing on the infection to family members when returning home. An ODP described that - despite observing precautions - the anxiety of passing on the infection to family members was a perpetual reality:

"We follow the precautionary measures while entering the hospital. We try to follow the same safety measures at home, so as not to get our family infected. But the fear of infecting family is always there. We cannot wash our breath like our clothes!" (IP14)

\section{Anxiety and religious faith}

Some participants remained anxious about remaining in ablution all the time, as they believed that their main form of protection against COVID-19 was prayer and piety. An ODP shared that since he believed it was impossible to maintain social distance and protective gear, he remained anxious about remaining in ablution at work:

"It is impossible to maintain social distance and protective gear. I have not got it [COVID-19] yet, and I believe it is because I stay Ba'Wudu [in the state of ritual ablution] at all times. My only worry is that my ablution does not break while at work." (IP4)

Another ODP reinforced that he worried about the short supply of protective gear, and so depended on God to protect him:

"There are shortages of protective gear. It is upsetting. But we are able to continue to work by putting our faith in Allah. Otherwise, things are very difficult. We can only pray for God to protect us." (IP5)

\section{Emotional health and stress}

Many participants shared a decline in mental health and increase in stress levels due to combined work pressure and the fear of infection:

"We have been doing a difficult and thankless job for years. There is now more mental load due to COVID19 safety precautions. Just mentally and physically we are tired... Nothing else." (IP37)

Another participant mentioned the emotional burden and overall stress of living with the pandemic:

“Before we were only taxed physically. Now there are emotions involved. We don't know about infected patients. We don't know if we will be well enough to come to work in coming days. We don't know if we are carrying the virus home. The stress levels are high all together." (IP7)

\section{Discussion}


The study results highlight the challenges for ODPs during the COVID-19 pandemic. Until now, there is no research focussing on this group of healthcare personnel in times of the COVID-19 pandemic available, although it is a vulnerable subgroup. ODPs are low to middle income earners and many have entered the profession with only secondary school degrees. Due to their underprivileged background, they are particularly vulnerable to infection burden from household locality. Within our study, ten themes were discovered which highlighted the problems that ODPs faced in delivering services and securing patient safety during the pandemic and otherwise.

The first finding was related to training for COVID-19. There was a misunderstanding that previous overall training for infectious disease control would be sufficient. Although no study participant had received any advanced training, some had at least a briefing from a senior health professional. Overall, there was reliance on social media and on-the-job experience for training. However, it is essential to provide extended COVID-19-specific training (e.g. hand washing procedure, donning and doffing protective gear, face mask guidelines) to ensure practitioner and patient safety [32]. Worldwide the most difficult issue for hospitals and health sectors has been the allocation of resources and time for training of healthcare practitioners (HCPs) [24], especially emergency surgical teams [33]. A large amount of ODPs within our study have received only secondary schooling. Thus, it is important that ODPs in Pakistan and other developing regions from lower educational backgrounds receive more support in continuous training and skill development [34]. For that reason, training for skill development and professional convergence is needed, along with a focus on non-technical skill development and team-building to improve communication and patient safety. Simulation training is encouraged for ODPs and surgical teams to avoid adverse events in emergency settings and support improved communication between practitioners coming from highly diverse skill backgrounds. Training for COVID-19 must not be limited to prevention and protective gear management, but also include updated information about epidemiology of and testing for the disease.

A second finding was with relation to COVID-19 testing. Globally, there were challenges with regard to emergency patients not being able to be tested in time and thus placing the theatre and hospital staff at risk of infection [35]. ODPs were not being supported with regular testing by the employer and had to pay for testing themselves and visit other hospitals for consultancy. In some cases, paying for COVID-19 testing amounted to one-third of the monthly salary of ODPs. Other recent research argues that unless healthcare staff are supported with easy access to diagnostics and medical support they will be unable to provide optimal services to critical patients seeking emergency care [36]. Another issue of concern was that ODPs had inadequate knowledge about tests results and believed that a negative result in nonspecific antibody tests (like lgG) indicated that lenient preventive protocols could be adopted. This is an issue of inadequate knowledge of tests, but also of non-standardized policies across hospital structures and practitioners [37].

A third finding was about the critical supply shortages and poor quality of PPE. Reasons for limited supply not only include shortages from suppliers and contractors, but also discrimination in supply by administration. Also previous research has highlighted that supply issues and shortages exist even in 
developed countries [38]. However, special attention is needed for the discrimination in distribution between HCPs in Pakistan and other developing regions. Such discrimination may be a result of status discrepancy between providers [39].

A fourth area was the challenge of maintaining physical distance and preventive protocols. The nature of the job and workplace routines for ODPs meant that they were unable to maintain physical distance from patients and co-workers. There were also issues of inability to secure complete cleaning of the theatre or maintain protective gear from patient to patient in emergencies due to a lack of time and high work burden. ODPs also highlighted that in some hospitals no modifications in protocols for disinfection and sterilization have been adopted since the COVID-19 pandemic. Recent research confirms that complete and appropriate use of PPE kits is essential for HCPs who cannot avoid frequent and close physical contact with patients. Furthermore, there should be enough staffing and time for preventive practices [40]. Proper knowledge of different processes and agents for sterilization and disinfection are essential to secure patient safety in the theatre [32]. Sterilization and disinfection protocols must be standardized and made consistent across hospitals settings.

A fifth area was that of shortages in human resources and role burden of ODPs. It was highlighted that there is a serious shortage of ODPs, which leads to additional work burden and lack of time for prevention during the pandemic. There was no extra remuneration for the additional responsibilities allocated during the pandemic for disinfection and sterilization. It has been emphasized that equitable compensation of HCPs leads to an improvement in job satisfaction and patient care [41]. We also found that in some cases COVID-19 positive ODPs who remained asymptomatic were asked by the employer to continue work due to shortages. Increasing ODP staffing in the theatre has advantages of assisting in the distribution of responsibility, cooperation in processing information related to the patient, and managing infection control [42].

A sixth finding is that of problems with hospital administration. ODPs are unable to communicate with the administration or solicit a response from them as they were considered non-significant team members. Research suggests that health service quality and patient safety is dependent on efficient and responsive hospital administration [43]. We also found that ODPs are hampered by the unsafe planning of theatre design and lack of safe avenues for waste disposal. Design of the operation theatre and waste disposal paths are critical supportive characteristics needed to build an environment of safety [44]. There is need for immediate re-construction of theatres to provide avenues for waste disposal and prevention of cross-contamination. Waiting areas for family must be located at a reasonable distance and overall random construction must be prohibited in the future while observing international recommendations for theatre construction and patient safety. ODPs also levelled criticism about the turning away of critical obstetric patients who were not registered with the hospital, which has implications for maternal and new-born safety. Local research confirms that the public health sector needs more funding and capacity to cater to the need of critical obstetric patients from primary to tertiary sector [45]. 
A seventh theme of problems related to exclusion and hierarchy. ODPs complained about a lack of inclusion in matters of governance and compensation. Research suggests that ODPs and AHPs who are not provided involvement in governance are less likely in assuming responsibility for patient safety [46]. Local research confirms that AHPs in Pakistan, despite being regular government employees, have unsatisfactory salaries and employment benefits [47]. The inferior position of ODPs was described through examples of discrimination in supply of protective gear and training opportunities, in comparison to colleagues like physicians and nurses. Other literature corroborates that ODPs across the world suffer from exclusion due to their historical enlistment as untrained support staff [48]. ODPs are also known to suffer from long-standing power politics and hierarchal bullying in the theatre, with the surgeons and anaesthesiologists on the top, the nurses in next line, and the ODPs last [14].

The eighth theme was of problems related to teamwork and communication. ODPs faced coordination barriers with pre- and post-operative as well as intra-operative teams. Study participants also complained that they did not receive collective training with the surgical team preventing team-building. Patient safety in the surgical setting is highly dependent on the non-technical aspects of theatre processes, such as team development and communication skills [49]. In fact, some research argues that evidence-based best practices for infection prevention are less valuable, compared to collaboration and communication between practitioners [50]. Therefore, regular team briefing and consultation meetings between the entire surgical team and administration are necessary to secure perioperative safety. Issues of hierarchy and lack of communication must be resolved, through team-building workshops and punitive measures against workplace bullies, in order to encourage sharing of information for patient safety.

There were also issues of poor communication with patients from various backgrounds and the inability to manage family attendants and even face aggression from them. Language and literacy barriers with patients from various socio-demographic backgrounds can contribute to adverse events [51]. Although the number of visiting family attendants has been limited to one during COVID-19, informal cultural norms and fear of surgical errors, malpractice or negligence drive multiple family members to accompany patients in hospitals of Pakistan [52]. Furthermore, aggression by family attendants during COVID-19 may have augmented due to: (i) fear of inadequate sterilization maintenance, and (ii) belief that the government is falsely diagnosing people as COVID-19 positive, and in some cases even killing them, to receive more funding [53].

The ninth theme of problems related to error management. ODPs highlighted the types of errors including breaches during emergency surgeries due to work overburden, inability to maintain protective gear in emergency and hurried circumstances, and failure to understand surgeon instructions. Errors and efficiencies are grounded in system and administrative limitations [54]. In Pakistan, there is no error reporting system for protocol violation or surgical error for ODPs or other hospital team members [55]. ODPs in the study rationalized that most errors were unintentional and due to the nature of the job. International literature advocates that there is need for constant monitoring and strategy development to prevent situational violations during surgery [56]. ODPs also maintained that they were unable to share errors and safety concerns with doctors and management. Other research confirms that junior

Page 20/26 
practitioners are unable to share patient safety concerns and errors due to fears of job loss and litigation [57].

The tenth and final theme related to anxiety and fear. ODPs remained in fear of getting infected with COVID-19 despite safety measures, catching the virus in the closed setting of the theatre, and the close proximity of theatre staff and patients. There was also fear of carrying the virus home and passing it to family members. These fears are also linked to adverse effects on mental health. Other local research confirms that healthcare staff is facing significant mental health challenges due to the pandemic [58]. Other research verifies that ODPs can suffer from stress and perceptions of inefficiencies due to interprofessional failure and lack of teamwork in the theatre [14]. The anxiety about contracting COVID-19 and shortage in preventive gear supply or maintenance has led to reliance on religious beliefs for protection. However, misconceptions can encourage stigma, discrimination and lapses in preventive protocols [59].

\section{Limitations}

We were successful in interviewing ODPs working in emergency surgeries during the COVID-19 pandemic. The limitations of this study include perception-based responses and a small sample size. We were also only able to gain permission to sample public sector teaching hospitals in Lahore. However, the advantage is that all sampled hospitals are regulated by the same health sector body, which prevents problems of differences in comparison. Furthermore, it is the first study of its kind to investigate the concerns of an invisible health workforce, who are a fountain of information regarding the closed surgical theatre environment. This study has also helped to identify critical gaps in the delivery of emergency surgical services and infection control during pandemics. We believe this study has presented salient qualitative findings to help generate hypotheses about practices that need improvement in government hospital settings and future research can now target hypothesis testing in representative quantitative studies.

\section{Conclusions}

We identify ODPs as the heart of the theatre, because they are key agents in supporting emergency surgeries during the pandemic. However, ODPs are faced with several challenges in times of COVID-19 such as little training, professional development, and resource shortages. Globally, ODPs are in need of urgent support to improve their professional identity in the theatre and in the hospital setting generally [18]. This mobilization for change has to come from health sector bodies responsible for licensing, regulation, training, and skill development of ODPs, along with hospital administration and professional colleagues. Apart from the concerns for patient safety and provider job satisfaction, improved regulation and licensing of ODPs, and other providers, is important to prevent quackery and perpetration of other crimes. For instance, male ward boys and theatre attendants have been listed as perpetrators of sexual harassment crimes against both female practitioners and female patients in the healthcare settings [60]. 
We also conclude that ODPs are key players in sharing information related to improvement in surgical practices.

\section{Abbreviations}

AHP Allied health professionals

HCP Healthcare practitioner

IP Interview partner

ODP Operating department practitioner

OTA Operating theatre assistant

PKR Pakistani Rupee

PPE Personal protective equipment

USD US-Dollar

\section{Declarations}

\section{Ethical approval and consent to participate}

Ethical approval was taken from King Edward Medical University. Written informed consent was taken from each participant, for both participation in the study and audio recording of telephonic interviews. Participants were not provided any incentive to take part in the study.

\section{Consent for publication}

Not applicable.

\section{Availability of data and materials}

Data is available from corresponding author upon reasonable request.

\section{Conflicts of interest}

There is no conflict of interest to declare.

\section{Funding}

No funding was received for this study.

\section{Authors' contributions}


SRJ designed this study, analysed the data and prepared the manuscript. AM and NM collected the data and assisted in data analysis. SAN provided contacts for sampling. FF supervised the research process. AM, NM, SAN and FF revised the manuscript for important intellectual content. All authors approved the final version of this manuscript.

\section{Acknowledgements}

We thank all the ODPs who provided their time and willingness to participate in this study. We would like to thank Mr. Muhamamd Naeem (Coordinator, Punjab Medical Faculty), Dr. Zeeshan Sarwar (Associate Professor Surgery \& Incharge OTAs at Mayo Hospital Lahore), and Dr. Fakeha Rehman (Assistant Professor Pathology King Edward Medical University, instructor Allied Health Sciences) for key information about ODP recruitment, licensing, and training. We also thank the three currently working ODPs not part of this study who gave us so much time to help confirm our findings. Finally, we would like to acknowledge our research assistants Ms. Noor ul Ain, Mr. Mir Mohammad Bezinjo, and Ms. Faroza Butt for their assistance in transcription from audio recordings to Microsoft Word and then transfer to NVIVO.

We acknowledge support from the German Research Foundation (DFG) and the Open Access Publication Fund of Charité - Universitätsmedizin Berlin.

\section{References}

1. Mawalla $B$ et al.,Predictors of surgical site infections among patients undergoing major surgery at Bugando Medical Centre in Northwestern Tanzania. BMC Surgery. 2011;11:21.

2. Allard J et al. "Who's on the team today?" The status of briefing amongst operating theatre practitioners in one UK hospital. Journal of Interprofessional Care. 2007;21(2): 189-206.

3. Li Y, Zeng L, Li Z et al. Emergency trauma care during the outbreak of corona virus disease 2019 (COVID-19) in China. World Journal of Emergency Surgery. 2020;15:1-10.

4. Fisha $\mathrm{K}$ et al. The prevalence and root causes of surgical site infections in public versus private hospitals in Ethiopia: a retrospective observational cohort study. Patient Safety in Surgery. 2019;13(1):26.

5. Allegranzi B, Pittet D. Healthcare-associated infection in developing countries: Simple solutions to meet complex challenges. JSTOR. 2007.

6. Spinelli A, Pellino G. COVID-19 pandemic: perspectives on an unfolding crisis. British Journal of Surgery. 2020.

7. Mai-Phan TA et al., Emergency room surgical workload in an inner city UK teaching hospital. World Journal of Emergency Surgery. 2008;3(1):19.

8. Bronsert MR et al. The value of the "Surgical Risk Preoperative Assessment System" (SURPAS) in preoperative consultation for elective surgery: a pilot study. Patient Safety in Surgery. 2020; 14(1):112. 
9. Byrne BE et al. Understanding how colorectal units achieve short length of stay: an interview survey among representative hospitals in England. Patient Safety in Surgery. 2015;9(1):1-6.

10. Wong SW, Smith R, Crowe P. Optimizing the operating theatre environment. ANZ Journal of Surgery. 2010; 80(12):917-24.

11. Berenguer $\mathrm{CM}$ et al. Improving surgical site infections: using National Surgical Quality Improvement Program data to institute Surgical Care Improvement Project protocols in improving surgical outcomes. Journal of the American College of Surgeons. 2010; 210(5): 737-41.

12. Wicker P. Caring for perioperative patients. Journal of Operating Department Practitioners. 2015; $3(1): 27-32$.

13. Rowe N. A discussion on research and appraisal within comparative perioperative professions: the potential contribution to ODP professional development. Technic-Journal of the College of Operating Department Practice. 2007;3(11):16-9.

14. Coe R, Gould D. Disagreement and aggression in the operating theatre. Journal of Advanced Nursing. 2008; 61(6):609-18.

15. Kilvington B. The History of the Association of ODPs. 1998.

16. Timmons S, Tanner J. A disputed occupational boundary: operating theatre nurses and operating department practitioners. Sociology of Health Illness. 2004;26(5):645-66.

17. Cadman V. Career opportunities for theatre practitioners. Journal of Perioperative Practice. 2017; 27(11):246-7.

18. Lowes R, Duxbury A, Garth A. The evolving roles of Operating Department Practitioners in contemporary healthcare: A service evaluation. Journal of Perioperative Practice. 2020; 30(3):46-56.

19. Sartelli $M$ et al. A proposal for a comprehensive approach to infections across the surgical pathway. World Journal of Emergency Surgery. 2020; 15(1):1-26.

20. Allard $\mathrm{J}$ et al. "Who's on the team today?" The status of briefing amongst operating theatre practitioners in one UK hospital. Journal of Interprofessional Care. 2007; 21(2):189-206.

21. Jafree SR. Workplace violence against women nurses working in two public sector hospitals of Lahore, Pakistan. Nursing Outlook. 2017; 65(4):420-7.

22. Kumar $\mathrm{R}$ et al. Correlates of knowledge, attitude and practices about health care waste management among hospital workers of Pakistan. Journal of Liaquat University of Medical Health Sciences. 2018; 17(1):1-7.

23. Raut R et al. Impact of COVID-19 on Neurosurgery in LMIC: Training and Service Delivery in a Tertiary Care Hospital in Pakistan. Indian Journal of Neurotrauma. 2020.

24. Høyland S, Aase K, Hollund JG. Exploring varieties of knowledge in safe work practices-an ethnographic study of surgical teams. Patient Safety in Surgery. 2011; 5(1):21.

25. Yule S, Flin R, Maran N. Safer surgery Analysing behaviour in the operating theatre. 2009.

26. Government of Punjab. Punjab Government Directory for Division and District Health Facilities. 2020; Available from: 
https://health.punjab.gov.pk/directory/reports/Division_and_district_wise_facilities.pdf.

27. McElroy L et al. Applying the WHO conceptual framework for the International Classification for Patient Safety to a surgical population. International Journal for Quality in Health Care. 2016; 28(2):166-74.

28. Weller SC et al. Open-ended interview questions and saturation. PloS One. 2018; 13(6):e0198606.

29. Leech NL, Onwuegbuzie AJ. Beyond constant comparison qualitative data analysis: Using NVivo. School Psychology Quarterly. 2011; 26(1):70.

30. Clarke V, Braun V, Hayfield N. Thematic analysis. J Qualitative psychology: A practical guide to research methods. 2015: 222-48.

31. Saunders B et al. Saturation in qualitative research: exploring its conceptualization and operationalization. Quality Quantity. 2018; 52(4):1893-907.

32. Patwardhan N, Kelkar U. Disinfection, sterilization and operation theater guidelines for dermatosurgical practitioners in India. Indian Journal of Dermatology, Venereology, Leprology. 2011; 77(1):83.

33. Martin $\mathrm{G}$ et al. Simulator Teaching for Operating Department Practitioners. Simulation in Healthcare. 2006; 1(3):196.

34. Pinto $\mathrm{M}$ et al. Improving the knowledge of Operating Theatre Assistants. Sri Lankan Journal of Anaesthesiology. 2009; 17:2.

35. Ford JS et al. Testing Asymptomatic Emergency Department Patients for Coronavirus of 2019 (COVID-19) in a Low Prevalence Region. Academic Emergency Medicine. 2020.

36. Bielicki JA et al. Monitoring approaches for health-care workers during the COVID-19 pandemic. Lancet Infectious Diseases. 2020.

37. Mitchell BG et al. Hospital infection control units: staffing, costs, and priorities. American Journal of Infection Control. 2015; 43(6):612-6.

38. Patel A et al. Personal protective equipment supply chain: lessons learned from recent public health emergency responses. Health Security. 2017; 15(3):244-52.

39. Özcan K, Özkara B, Kızıldağ D. Discrimination in health care industry: a research on public hospitals. Equality Diversity Inclusion. 2011.

40. Quintão VC et al. The anesthesiologist and COVID-19. Brazilian Journal of Anesthesiology. 2020.

41. Romig B, Maillet J, Denmark RM. Factors affecting allied health faculty job satisfaction A literature review. Journal of Allied Health. 2011; 40(1):3-14.

42. Patel VL et al. The collaborative health care team: the role of individual and group expertise. Teaching Learning in Medicine. 2000; 12(3):117-32.

43. Mitchell PH. Defining patient safety and quality care, in Patient safety and quality: An evidencebased handbook for nurses. Agency for Healthcare Research and Quality (US); 2008.

44. Pittet $D$, Donaldson L. Challenging the world: patient safety and health care-associated infection. International Journal for Quality in Health Care. 2006; 18(1):4-8. 
45. Ismail S, Sohaib M. Critically ill obstetric patients in resource-limited settings. Journal of Obstetric Anaesthesia Critical Care. 2019; 9(2):53.

46. Needle JJ, Petchey RP, Benson J, Scriven A, Lawrenson J, Hilari K. The role of allied health professionals in health promotion. NIHR Service Delivery and Organisation programme; 2011.

47. Hafeez A et al. Pakistan human resources for health assessment, 2009. Eastern Mediterranean Health Journal. 2010; 16:S145.

48. Gillespie BM, Pearson E. Perceptions of self-competence in theatre nurses and operating department practitioners. Journal of Perioperative Nursing in Australia. 2013; 26(1):29.

49. Friedman LH, Bernell S.L. The importance of team level tacit knowledge and related characteristics of high-performing health care teams. Health Care Management Review. 2006; 31(3):223-30.

50. Mitchell L, Flin R. Non-technical skills of the operating theatre scrub nurse: literature review. Journal of Advanced Nursing. 2008; 63(1):15-24.

51. Meuter RF, Gallois C, Segalowitz NS, Ryder AG, Hocking J. Overcoming language barriers in healthcare: A protocol for investigating safe and effective communication when patients or clinicians use a second language. BMC Health Services Research. 2015; 15:371.

52. Hashmi FK, Hashmi SK, Attitudes of attendants and visitors at the National Institute of Child Health (NICH): Problems and Solutions. Pak J Med Sci. 2006; 22(1):94-6.

53. Kermani S. Coronavirus: Rumours, fear and rising Covid deaths in Pakistan in BBC News. 2020.

54. Bleakley A. A common body of care: The ethics and politics of teamwork in the operating theater are inseparable. Journal of Medicine and Philosophy. 2006; 31(3):305-22.

55. Jafree SR, Zakar R, Zakar MZ, Fischer F. Assessing the patient safety culture and ward error reporting in public sector hospitals of Pakistan. Safety in Health. 2017; 3:10.

56. Sarker SK, Vincent C. Errors in surgery. International Journal of Surgery. 2005; 3(1):75-81.

57. Stahel PF, Flierl MA, Smith WR et al. Disclosure and reporting of surgical complications: a doubleedged sword?. American Journal of Medical Quality. 2010; 25(5):398-401.

58. Rana W, Mukhtar S, Mukhtar S. Mental health of medical workers in Pakistan during the pandemic COVID-19 outbreak. Asian Journal of Psychiatry. 2020; 51:102080.

59. Jafree SR, Naqi SA, Momina AU. Significant Other Family Members and Their Experiences of COVID19 in Pakistan: A Qualitative Study With Implications for Social Policy. American Psychological Association; 2020.

60. Jafree SR. Women, healthcare, and violence in Pakistan. Oxford University Press; 2018.

\section{Supplementary Files}

This is a list of supplementary files associated with this preprint. Click to download.

- SupplementaryAppendix 1 Interviewguide.pdf 